\title{
Research Paper: \\ Relationship of Leisure Time Activities With Fatigue and Mental Health Problems in People With Multiple Sclerosi
}

\author{
Seyed Mohammad Sadegh Hosseini ${ }^{1}$ (i), ${ }^{*}$ Sahar Nurani Gharaborghe ${ }^{1}$ (다
}

1. Department of Occupational Therapy, Faculty of Rehabilitation Sciences, Hamadan University of Medical Sciences, Hamadan, Iran.

\begin{tabular}{|c|c|}
\hline $\begin{array}{l}\text { Use your device to scan } \\
\text { and read the article online }\end{array}$ & Ittation Hosseini SMS, Nurani Gharaborghe S. [Relationship of Leisure Time Activities With Fatigue and Mental Health \\
\hline $014 \square$ & $\begin{array}{l}\text { Problems in People With Multiple Sclerosi (Persian)]. Archives of Rehabilitation. 2021; 22(2):154-167. https://doi.org/10.32598/ } \\
\text { RJ.22.2.675.5 }\end{array}$ \\
\hline 口ify & doil'https://doi.org/10.32598/RJ.22.2.675.5 \\
\hline
\end{tabular}

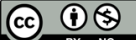

Received: 23 Apr 2020 Accepted: 15 Jan 2021 Available Online: $01 \mathrm{Jul} 2021$

Keywords:

Multiple sclerosis, Leisure time, Fatigue, Psychological disorders

\begin{abstract}
Objective One of the essential aspects of life in patients with Multiple Sclerosis (MS) is leisure time activities. One of the duties of occupational therapists is the evaluation and implementation of therapeutic interventions in the field of leisure for patients with neurological disorders such as MS. However, before presenting any intervention for increasing the ability of MS patients to spend their leisure time, it is necessary to study the concept of this field scientifically and systematically and identify and evaluate the factors affecting it. Many symptoms of MS, such as fatigue and mental health problems, may affect leisure activities. This study examines the relationship of leisure time activities with fatigue and mental health problems (stress, anxiety, and depression) in MS patients.

Materials \& Methods This research is a cross-sectional study. The study population consisted of all MS patients referred to Imam Khomeini Clinic, Mobasher Kashani Hospital, and MS Association in Hamadan City, Iran, in 2019. Of them, 99 (70 women, 29 men) with a Mean \pm SD age of 32.28 \pm 8.26 years were selected by using a convenience sampling method and based on the inclusion and exclusion criteria. For data collection, we used the MS leisure questionnaire, Fatigue Severity Scale (FSS), Visual Analog Fatigue Scale (VAFS), Expanded Disability Status Scale (EDSS), and Depression-Anxiety-Stress Scale (DASS). After obtaining written informed consent from the patients, the study questionnaires were completed by them. The collected data were analyzed in SPSS v. 16. The Spearman correlation test was used to examine the relationship of leisure time activities with fatigue and mental health problems.

Results There was a significant relationship between leisure activities and fatigue tests of VAFS $(P=0.003)$ and FSS $(P=0.001)$. Fatigue showed a high negative correlation with all leisure domains $(r=0.350, P=0.001)$ of difficult, social, spiritual/religious, out-of-home physical, and art/cultural activities and reduced them. The overall score of DASS was significantly correlated with leisure time spiritual/religious activity ( $r=$ -0.263), out-of-home physical activity ( $r=-0.213$ ) and art/cultural activity $(r=-0.205)$. Regarding its subscales, anxiety showed a significant correlation only with leisure time social activities $(r=-0.259)$, stress with spiritual/religious activities $(r=-0.212)$, and depression with all domains of leisure time activities except for difficult activities ( $\mathrm{P} \leq 0.005)$

Conclusion Leisure time activities are associated with fatigue and mental health problems (stress, anxiety, and depression) in MS patients. Stress and anxiety only affect their leisure time social activities. Fatigue or mental health problems can reduce the amount of leisure time.
\end{abstract}

\section{${ }^{*}$ Corresponding Author:}

Sahar Nurani Gharaborghe, MSc.

Address: Department of Occupational Therapy, Faculty of Rehabilitation Sciences, Hamadan University of Medical Sciences, Hamadan, Iran Tel: +98 (813) 8381571

E-Mail: saharnurani@yahoo.com 


\section{Extended Abstract}

\section{Introduction}

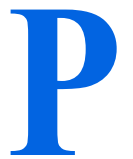

hysical, psychological, and social symptoms of Multiple Sclerosis (MS) can affect different aspects of life in MS patients. It can disrupt the work-related areas, including instrumental daily living activities, job, education, social participation, and leisure activities. Mood disorders are seen in more than $50 \%$ of these patients, of which depression is the most common and can occur in both primary and secondary ways reducing the quality of life in these patients. The probability of suicide in these patients is seven times higher than in healthy individuals [1]. Its causes can be physiological in response to the disease process, psychological in response to the diagnosis, or side effects of drugs that can be associated with fatigue and inability to cope and adapt [2]. Fatigue is the most common symptom reported by MS patients, which affects their progress of cognitive and physical therapy. Fatigue is defined as "a subjective lack of physical and or mental energy that is perceived by the individual or caregiver to interfere with usual and desired activities" [3, 4]. In a study on the effect of depression, fatigue, and cognitive dysfunction on the leisure activities of MS patients using the Nottingham leisure questionnaire, Hosseini et al. reported the effect of depression and fatigue but cognitive dysfunction on leisure [9]. A review of research conducted in Iran indicates that most researchers pay attention to the time spent on each leisure activity [6-9], while a few of these studies have examined the relationship of leisure time with mental and social health [10], social capital, social duty, leisure satisfaction, gender [11], number of children, level of education and physical activity [9].

In an epidemiological study with a large sample, Chwastiak et al. found a strong association between depression and MS severity, but no such association was found between depression and disease progression pattern. They suggested that newly diagnosed patients, patients with extensive functional changes, and those with limited social support should also be evaluated for depression [12]. One of the fields of work in occupational therapists is the evaluation and implementation of therapeutic interventions in the field of leisure for patients with neurological disorders such as MS [13]. However, before presenting any intervention for increasing the ability of MS patients to spend their leisure time, it is necessary to study the concept of this field scientifically and systematically and identify and evaluate its affecting factors. In this way, therapists can help increase their leisure time with appropriate therapeutic interventions. This study investigates the relationship of leisure activities with fatigue and mental health problems (stress, anxiety, and depression) in MS patients. A leisure questionnaire for Iranian MS patients with 5 domains was used, which has not been used in other related studies.

\section{Materials and Methods}

This research is a cross-sectional study. The study population consisted of all MS patients referred to Imam Khomeini Clinic, Mobasher Kashani Hospital, and MS Association in Hamedan City, Iran. For sampling, a convenience sampling method was used. The inclusion criteria were the definitive diagnosis of MS by a neurologist, ability to speak and communicate, and willingness to participate in the study. The exclusion criteria were having other diseases associated with MS, according to the neurologist, and unwillingness to continue participation. According to the Cochran formula and considering $\mathrm{Z}=1.96, \mathrm{p}=0.5, \mathrm{q}=0.5$, and $\mathrm{d}=0.1$, the sample size was obtained 96.

Data collection tools were first the leisure questionnaire for Iranian MS patients, developed by Hosseini et al. [19]. It has 50 items and 5 subscales of difficult activities, social activities, out-of-home physical activities, art/cultural activities, and spiritual/religious activities. The items are rated on a 4-point Likert-type scale. The validity coefficients for the single measure and average measure of this questionnaire in the pretest phase were 0.826 and 0.905 , respectively, and were statistically significant. This result indicates excellent internal consistency between the scores of subscales [20]. The second tool is the Depression, Anxiety, and Stress Scale (DASS) [20]. Each subscale of this tool has 7 items. The final score of DASS is obtained by summing up the scores of each subscale. Each item is rated from $0=$ "Did not apply to me at all" to 3= "applied to me very much" [20]. The test-retest reliability for the Persian version of DASS is 0.82 [21]. The third tool is the Fatigue Severity Scale (FSS), which was developed by Krupp et al. [23] for use in patients with systemic lupus and MS, including a Visual Analog Fatigue Scale (VAFS) and Expanded Disability Status Scale (EDSS). It has 9 items extracted from the 28 items of the fatigue questionnaire. The validity and reliability of the Persian version of this questionnaire were evaluated by Azimian et al. [24], who reported an Intraclass Correlation Coefficient (ICC) value of 0.93 .

After obtaining written informed consent from the participants, the questionnaires were distributed among them in 2019. After collecting data, they were analyzed in SPSS v. 16 software. The Spearman correlation test was used to examine the relationship between the dependent variable (leisure) and two independent variables (fatigue and mental health problems) after examining the normality of data 
Table 1. Demographic characteristics of the study participants $(n=99)$

\begin{tabular}{|c|c|c|c|c|c|}
\hline \multicolumn{2}{|r|}{ Variable } & \multirow{3}{*}{$\begin{array}{l}\text { No. (\%) } \\
70(70.7)\end{array}$} & \multicolumn{2}{|c|}{ Variable } & \multirow{2}{*}{$\begin{array}{l}\text { No. (\%) } \\
45(45.5)\end{array}$} \\
\hline \multirow{6}{*}{ Gender } & & & & Single & \\
\hline & Female & & & & \\
\hline & & & & Married & $50(50.5)$ \\
\hline & \multirow{3}{*}{ Male } & & Marital status & & \\
\hline & & ובר & & Divorced & $1(1.0)$ \\
\hline & & & & Widow, widower & $3(3.0)$ \\
\hline \multirow{5}{*}{ Education } & Illiterate & $1(1.0)$ & & Employed & $27(27.3)$ \\
\hline & No high school diploma & $13(13.1)$ & & Unemployed & $25(25.2)$ \\
\hline & High school diploma & $36(36.4)$ & Employment status & Retired & $5(5.0)$ \\
\hline & Bachelor's degree & $37(37.4)$ & & Housekeeper & $27(27.3)$ \\
\hline & Higher academic degree & $12(12.1)$ & & College student & 15(15.2) \\
\hline
\end{tabular}

Rehabilitation

distribution. The Chi-square test was used to examine the relationship between leisure activities and demographic characteristics that are nominal variables.

\section{Results}

Demographic information of the participants is presented in Table 1. They were 70 females and 29 males, most of whom were married. Most were employed $(n=27)$ or housekeepers $(n=27)$. They had a Mean \pm SD age of $32.28 \pm 8.26$ years with a Mean \pm SD disease duration of $6.83 \pm 5.63$ years. The results of the Chi-square test showed a significant relationship between leisure activities and education level $(\mathrm{P}=0.003)$, but other demographic variables had no relationship with leisure activities (Table 2). The results of the Spearman correlation test (Table 3 ) showed that both VAFS $(\mathrm{P}=0.003)$ and $\mathrm{FSS}(\mathrm{P}=0.001)$ scores had a significant relationship with leisure activities. Moreover, leisure activities showed a significant relationship with the overall DASS score $(\mathrm{P}=0.007)$ and its depression subscale $(\mathrm{P}=0.001)$, but not with the subscales of anxiety $(\mathrm{P}=0.780)$ and stress
$(\mathrm{P}=0.060)$. The EDSS score also had a significant relationship with leisure activities $(\mathrm{P}=0.001)$. Age $(\mathrm{P}=0.335)$ and duration of disease $(\mathrm{P}=0.297)$ had no significant relationship with leisure activities.

To examine the relationship of each five domains of the leisure questionnaire with fatigue and mental health problems, we used the Spearman correlation test. As shown in Table 4, the VAFS score showed a significant relationship with social activities $(\mathrm{r}=0.376)$, out-of-home physical activities $(\mathrm{r}=0.202)$ and art/cultural activities $(\mathrm{r}=0.186)$. The FSS score showed a high correlation with all five domains of leisure activities. The overall DASS score was significantly associated with spiritual/religious activities $(\mathrm{r}=0.263)$, outof-home physical activities ( $\mathrm{r}=0.213$ ), and art/cultural activities ( $\mathrm{r}=0.205)$. Regarding DASS subscales, the results showed that anxiety had a significant correlation only with social activities $(\mathrm{r}=0.259)$, stress with spiritual/religious activities $(\mathrm{r}=0.212)$, and depression with all leisure activities, except with difficult activities $(\mathrm{P}<0.005)$.

Table 2. The Chi-square test results for examining the relationship between leisure activities and demographic characteristics

\begin{tabular}{ccccc}
\hline Variables & df & $\boldsymbol{\chi}^{2}$ & Sig. & 0.743 \\
\hline Marital status & 159 & 147.02 & 0.938 \\
\hline Employment status & 265 & 230.52 & 273.73 & 0.003 \\
\hline Education & 212 & 59.58 & 0.249 \\
\hline Gender & 53 & & Archives of
\end{tabular}


Table 3. The Spearman correlation test results for examining the relationship between leisure activities and study variables $(n=99)$

\begin{tabular}{ccc}
\hline Variable & Correlation Coefficient & Sig. \\
\hline VAFS & -0.292 & 0.0001 \\
\hline FSS & -0.350 & 0.001 \\
\hline Stress & -0.190 & 0.060 \\
\hline Anxiety & -0.178 & 0.780 \\
\hline Depression & -0.332 & 0.001 \\
\hline DASS & -0.269 & 0.007 \\
\hline EDSS & -0.925 & 0.001 \\
\hline Age & -0.098 & 0.335 \\
\hline Disease duration & -0.106 & 0.297 \\
\hline
\end{tabular}

DASS: Depression-Anxiety-Stress Scale; EDSS: Expanded Disability Status Scale; VAFS: Visual Analog Fatigue Scale; FSS, Fatigue Severity Scale.

\section{Discussion and Conclusion}

This study's findings revealed a significant relationship between leisure activities with fatigue and mental health problems in MS patients. No correlation was found between leisure activities and the patient's age, indicating that leisure time does not change with aging, which may be reasonable. People at any age have their own hobbies and the total leisure time amount is the same. No correlation was found between leisure time and disease duration, although it seems that as the duration of the disease increases, the rate of disability increases, which can affect the amount of leisure time. This issue is probably because the patients try to maintain their leisure time by doing other activities. Gender had no significant relationship with leisure activities; i.e., there was no difference between men and women in the amount of leisure time. Employment status and marital status did not affect the amount of leisure time, either. It means that the MS patients, whether married, single, employed, or unemployed, have a comparable amount of leisure time. Education level, however, showed a significant relationship with leisure activities. A person with a higher level of edu-

Table 4. Relationship of five domains of leisure activities with fatigue and mental health problems

\begin{tabular}{|c|c|c|c|c|c|c|c|c|c|c|}
\hline \multirow{2}{*}{ Variable } & \multicolumn{2}{|c|}{ Domain 1} & \multicolumn{2}{|c|}{ Domain 2} & \multicolumn{2}{|c|}{ Domain 3} & \multicolumn{2}{|c|}{ Domain 4} & \multicolumn{2}{|c|}{ Domain 5} \\
\hline & $\begin{array}{l}\text { Correlation } \\
\text { Coefficient }\end{array}$ & Sig. & $\begin{array}{l}\text { Correlation } \\
\text { Coefficient }\end{array}$ & Sig. & $\begin{array}{l}\text { Correlation } \\
\text { Coefficient }\end{array}$ & Sig. & $\begin{array}{l}\text { Correlation } \\
\text { Coefficient }\end{array}$ & Sig. & $\begin{array}{l}\text { Correlation } \\
\text { Coefficient }\end{array}$ & Sig. \\
\hline VAFS & -0.118 & 0.243 & -0.376 & 0.001 & -0.137 & 0.178 & -0.202 & 0.045 & -0.186 & 0.0001 \\
\hline FSS & -0.245 & 0.045 & -0.0001 & 0.003 & -0.224 & 0.026 & -0.260 & 0.009 & -0.301 & 0.002 \\
\hline Stress & -0.074 & 0.464 & -0.115 & 0.258 & -0.212 & 0.035 & -0.196 & 0.052 & -0.172 & 0.088 \\
\hline Anxiety & -0.081 & 0.423 & -0.152 & 0.134 & -0.259 & 0.010 & -0.180 & 0.074 & -0.044 & 0.668 \\
\hline $\begin{array}{l}\text { Depres- } \\
\text { sion }\end{array}$ & -0.196 & 0.052 & -0.00 & 0.048 & -0.289 & 0.004 & -0.287 & 0.004 & -0.303 & 0.002 \\
\hline ADSS & -0.157 & 0.121 & -0.190 & 0.060 & -0.263 & 0.009 & -0.213 & 0.035 & -0.205 & 0.042 \\
\hline EDSS & -0.731 & 0.001 & -0.768 & 0.001 & -0.369 & 0.001 & -0.704 & 0.001 & -0.529 & 0.001 \\
\hline
\end{tabular}

DASS: Depression-Anxiety-Stress Scale; EDSS: Expanded Disability Status Scale; VAFS: Visual Analog Fatigue Scale; FSS: Fatigue Severity Scale. 
cation has more fun than an illiterate person because educated people have more information about recreation and its effect on the disease.

Fatigue showed a high correlation with all domains of leisure activities. That is, fatigue affects difficult, social, spiritual/religious, out-of-home, and art/cultural activities. Overall, it can reduce the amount of all types of leisure activities. This finding is reasonable because the tired person cannot do leisure activities and prefers to rest more rather than doing something. This finding is consistent with the results of Hosseini et al. [9], who used the Nottingham leisure questionnaire. Khemthong et al. [25] concluded that social leisure time affects the physical health of MS women with fatigue. The stress and anxiety of MS patients in our study only affected their social leisure activities.

Janssens et al. [26] reported that stress and anxiety exist in MS patients since the onset of the disease. Brown et al. showed that depression leads to fatigue and anxiety in MS patients and vice versa [27]. In our study, depression affected all domains of leisure time in MS patients. Numerous studies have examined the relationship between leisure and depression [5, 23]. Ben Ari et al. concluded that depression affects participation in daily life in MS patients [20]. Molt et al. showed that depression and fatigue affect patients' leisure time physical activities [25]. In a longitudinal study, Stephens et al. showed that having moderate to severe leisure time physical activities can reduce depression and fatigue in MS patients [26]. No study was found on examining leisure domains among people with MS, but some studies link the physical domain of leisure to fatigue [27, 28]. For example, a study was found by Fjeldstad et al. [32] on the relationship between fatigue and the physical domain of leisure using the FSS and Godin leisure-time exercise questionnaire. Their results showed a significant relationship between the two variables. In a study by Vanner et al. [33], a significant relationship was also found between depression and physical leisure activities. These results are consistent with our findings. Fatigue is one of the most debilitating symptoms of MS, which affects the whole life of a patient.

One of the study's limitations was the small number of male patients compared to females, which seems acceptable given the proportion of these patients in the community, so the generalization of the results of the present study to men should be made with caution. It is recommended that the relationship between MS patients' leisure time and other aspects in Iran be investigated in future studies. Moreover, it is recommended to consider the effect of other symptoms of MS such as sleep problems, urinary and fecal control problems, and cognitive problems on the leisure time of MS patients.
Leisure time activities are associated with mental health problems and fatigue in people with MS. In other words, if MS patients suffer from disorders such as fatigue and mental health problems (depression, stress, and anxiety), it can reduce their amount of leisure time.

\section{Ethical Considerations}

\section{Compliance with ethical guidelines}

This study was approved by the Ethics Committee of Hamadan University of Medical Sciences (Code: IR.UMSHA.REC.1397.436)

\section{Funding}

This study was extracted from a research project approved by the Deputy for Research and Technology of Hamadan University of Medical Sciences (Code: 9708224844).

\section{Authors' contributions}

Both authors equally contributed to preparing this article.

Conflict of interest

The authors declared no conflict of interest. 


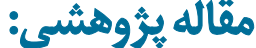 \\ ارتباط بين اوقات فراغت، خستكى و مشكلات روانى در افراد با مولتيبل اسكلروزيس
}

\author{
سيد محمدصادق حسينى' (0)، "سحر نورانى قرابرقع'
}

1. كروه آموزشى كاردرمانى، دانشكده علوم توانبخشى، دانشُّاه علوميزشكى همدان، هملان، ايران.

\begin{abstract}
حكبد
تاريخ دريافت: †.ارديبهشت

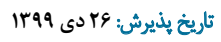

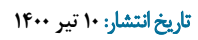

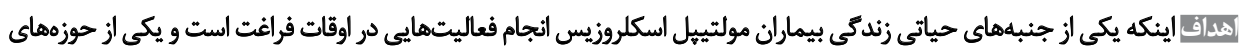

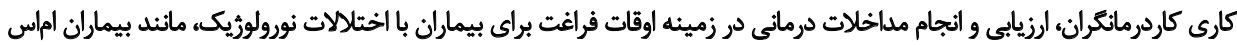

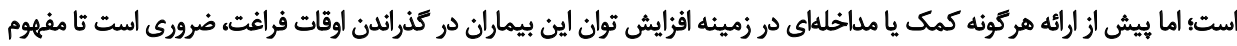

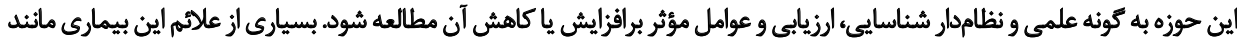

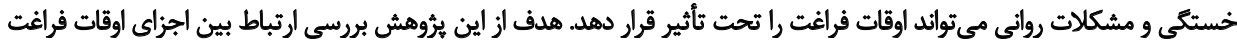

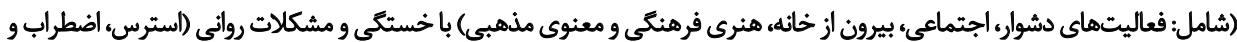

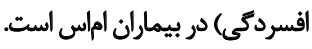

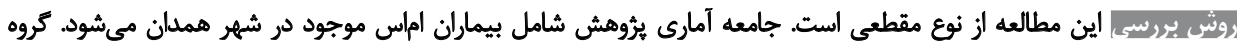

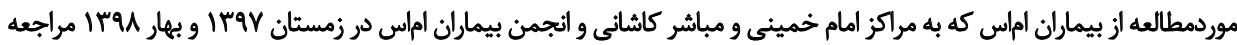

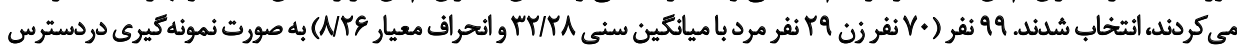

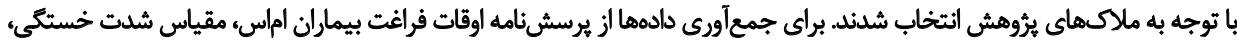

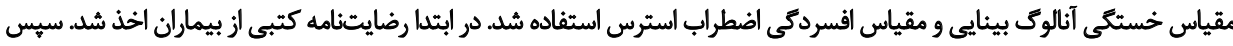

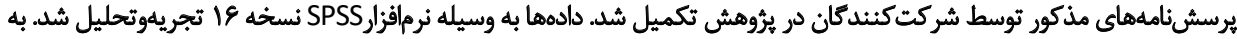

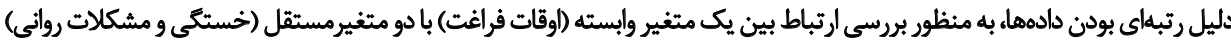

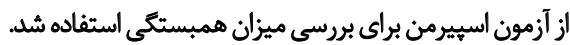

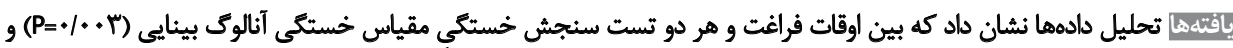

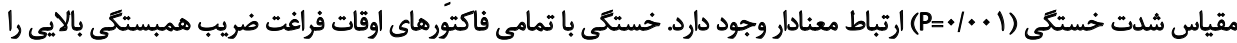

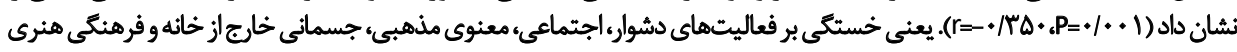

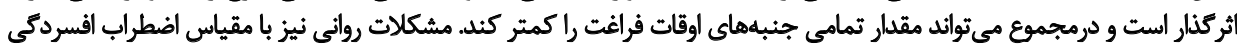

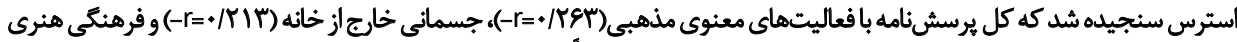

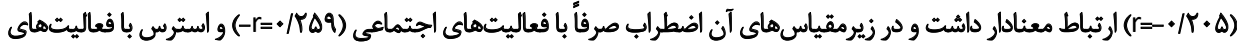

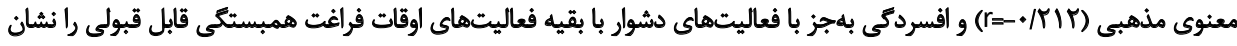

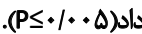

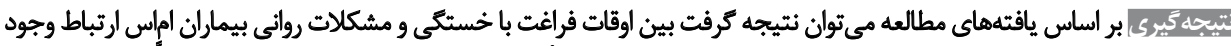

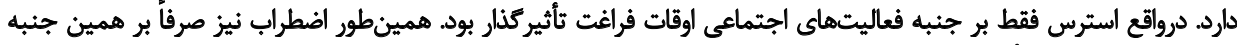

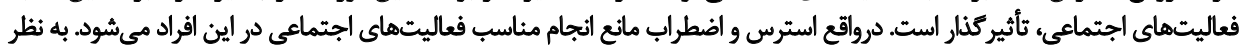

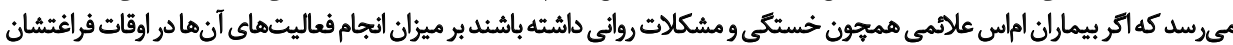
تأثير مي كذارد و مي تواند مقدار آن را كاهش دهد مند

كليدوازوها:

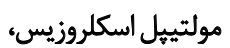

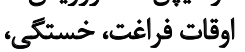

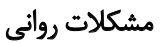


مستقيم با سلامت دارد و نخستين مورد از حيطههاى كارى

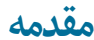

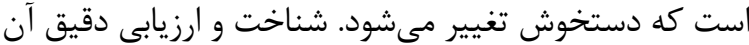

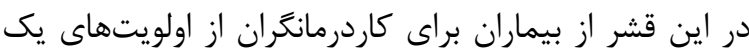

مداخله همهجانبه است.

باوجود اين دانش مادر زمينه شناخت و ارزيابى مشاركت به طور

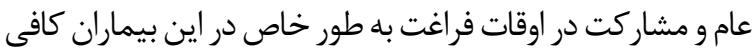

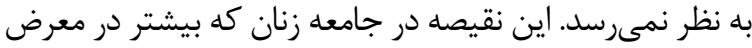

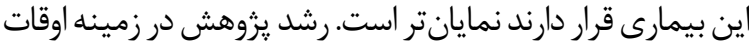

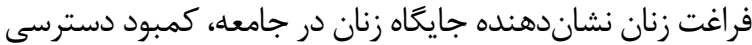

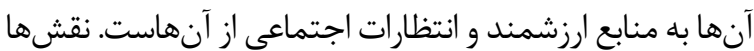

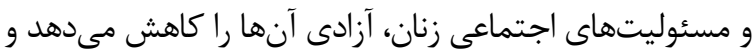

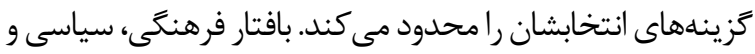

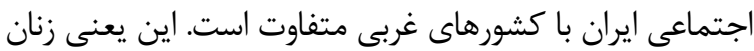

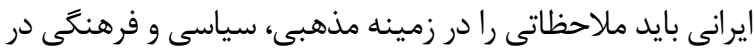

فعاليتهاى اوقات فراغت خود داشته باشند [. [1]

مرور يزوهشهاى انجامشده در ايران حاكى از آن است كه توجه

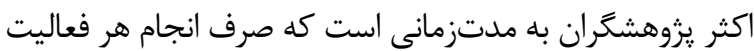

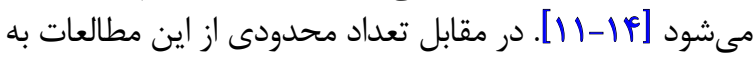

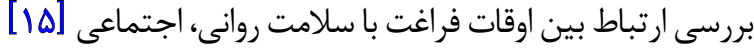

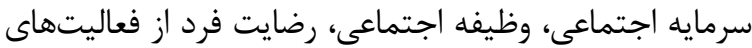

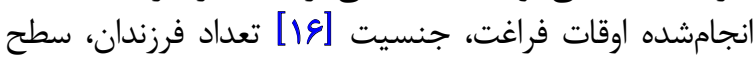

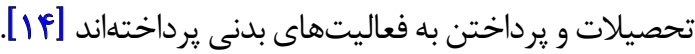
جواستياك و همكاران در يك يزوهش إيدميولوزيكى با نمونه

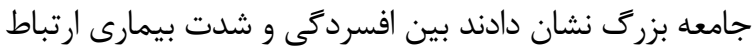

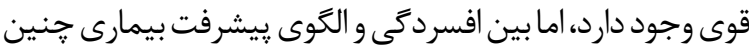

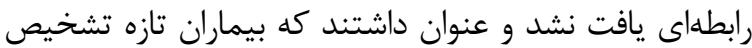

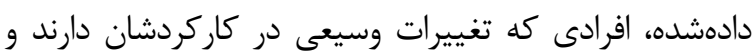

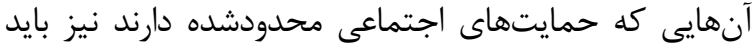

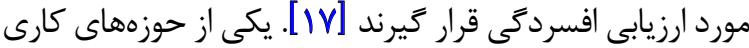

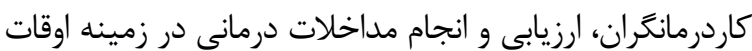

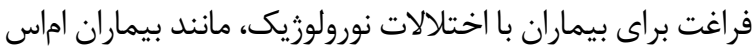

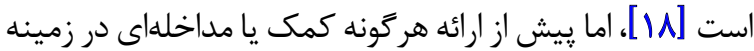

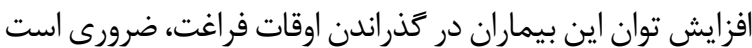

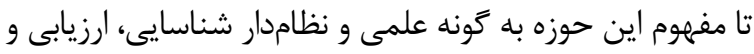

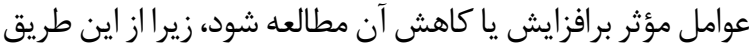

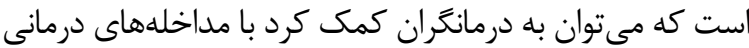

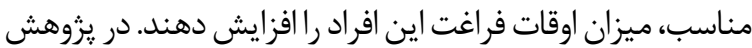

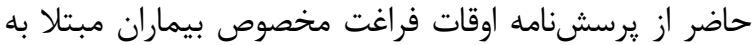

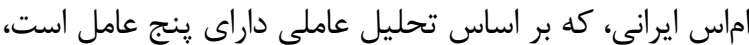

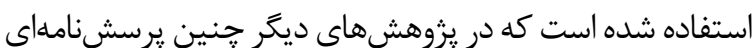

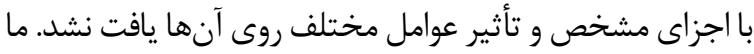

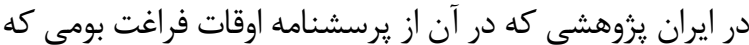
طبقدبندى شده باشد و از آن براى سنجش آن يرسئ ارتباط ديخر متغيرها

بيمارى مولتييل اسكلروزيس معمولاً در دوران جوانى آغاز

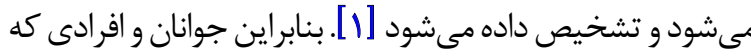

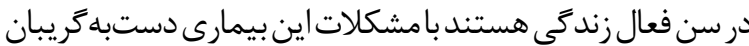

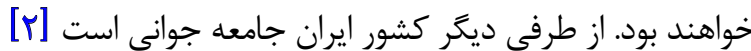

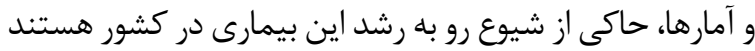

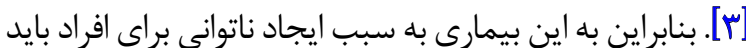

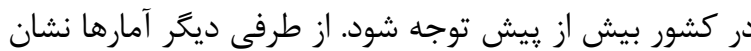

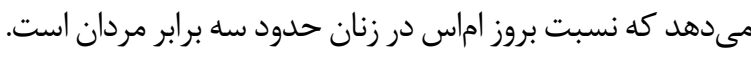
علائم جسمى، روانى و اجتماعى اين بيمارى در زند زتى ميى تواند

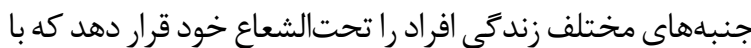

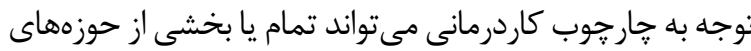

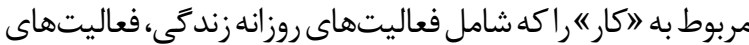

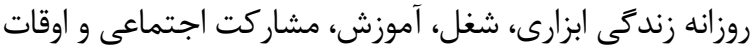

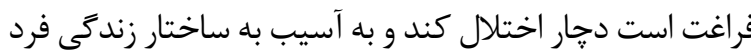
منجر شود و توازن كارى او را از بين ببرد.

اختلالات خلقى در بيش از • له درصد اين بيماران ديده مىشود

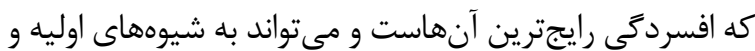

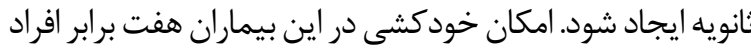

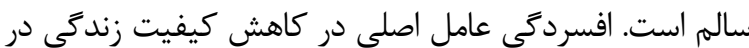

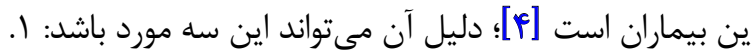

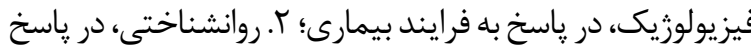

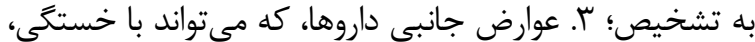
توانايى مقابله كردن و تطبيق ارتباط داشته باشد [هان]. خستكى معمولترين علامتى است كه بيماران اماس كزارش

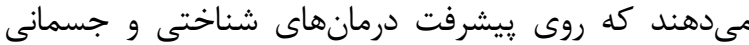

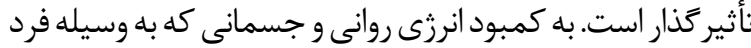

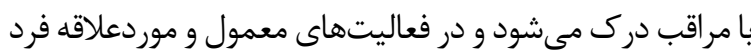

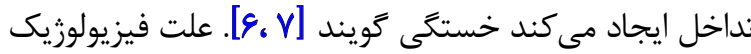

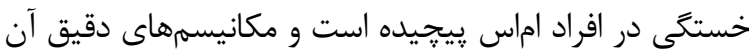

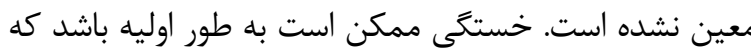

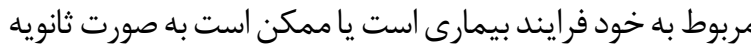

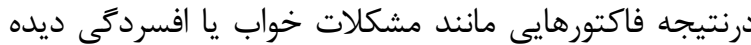

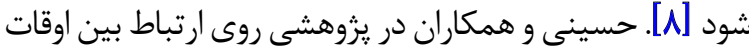

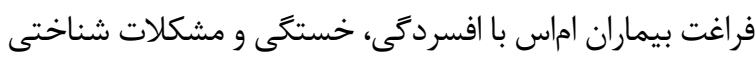

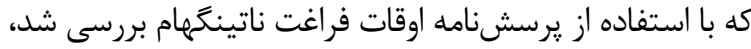

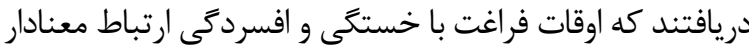

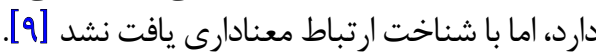
شناخت مشاركت و جنبههاى آن براى كاردرمانكَران اهميت

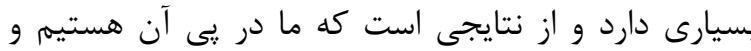

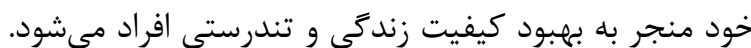

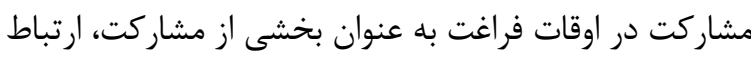




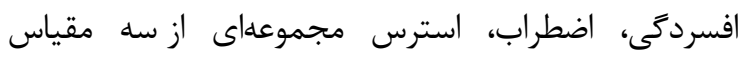

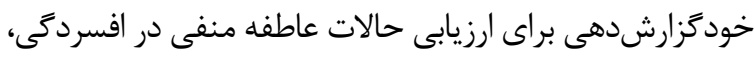

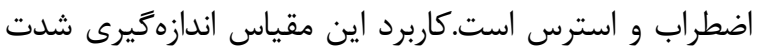

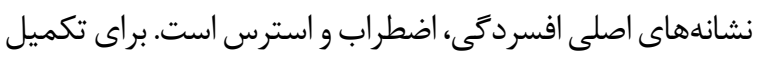

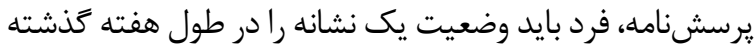

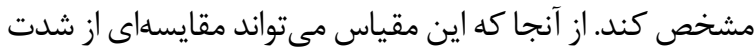

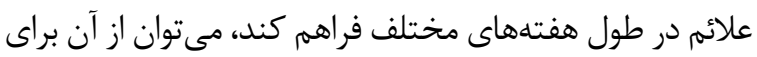

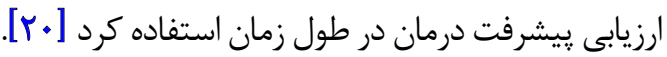
هريك از خردهمقياسهاى DASS شامل هفت سؤال است كه

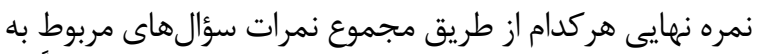

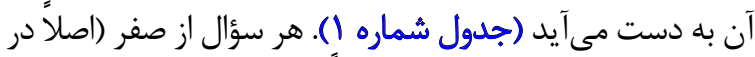

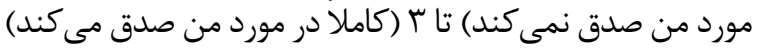

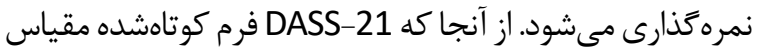

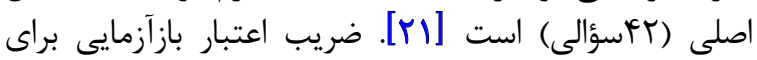

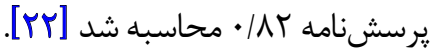

مقياس شدت خستخىى: مقياس سنجش شدت خستخى كه

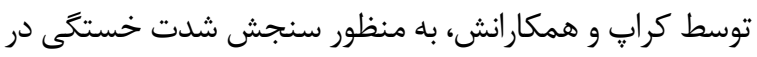

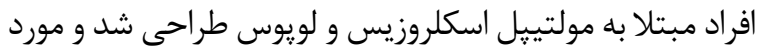

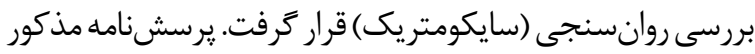

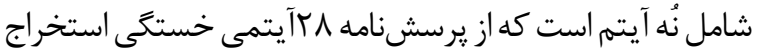

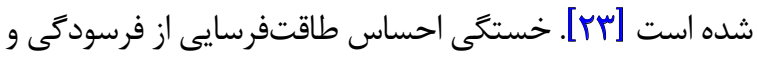

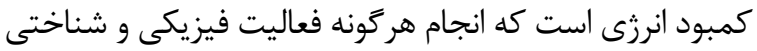

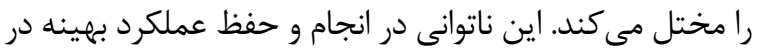

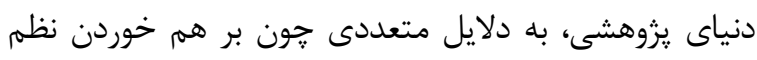

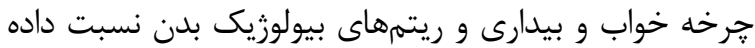

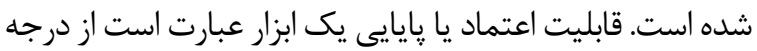

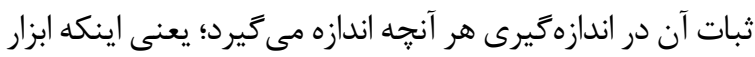

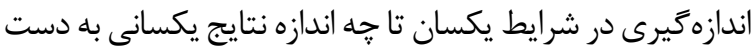

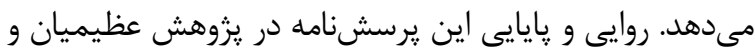

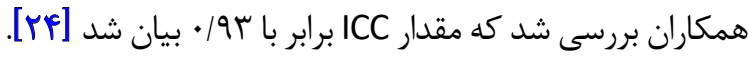

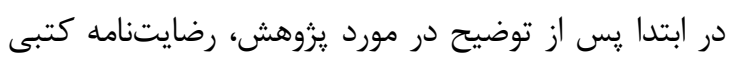

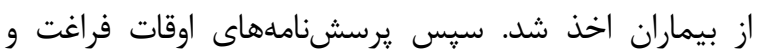

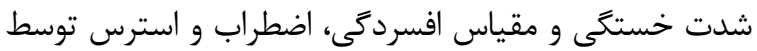

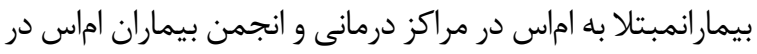

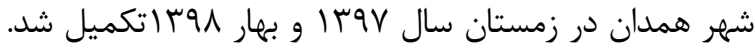

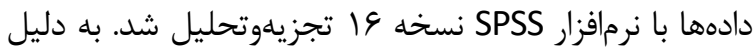

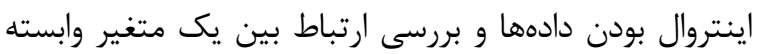

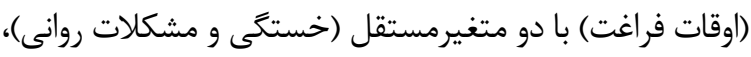

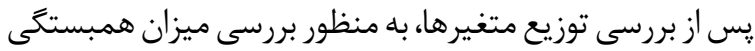

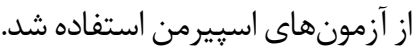

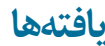

|طلاعات جمعيتشناختى شركت كنند
با اوقات فراغت يرداخته باشد نيافتيم، از اين رو هدف از اين

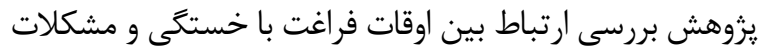
روانى (استرس، اضطراب و افسردگى) در بيماران اماس است.

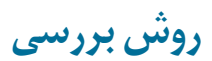

اين مطالعه از نوع مقطعى است. جامعه آمارى يزوهش شامل

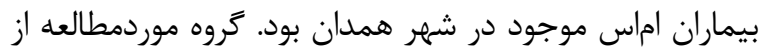

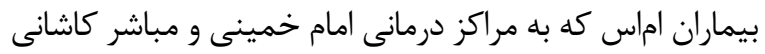

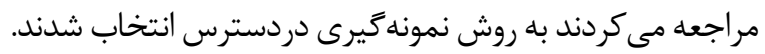

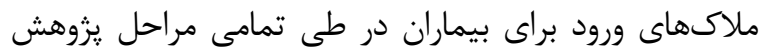

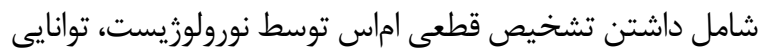

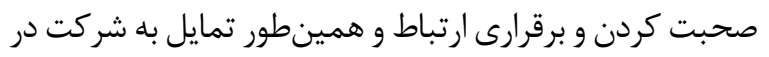

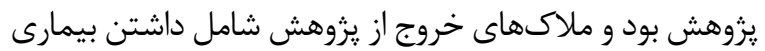

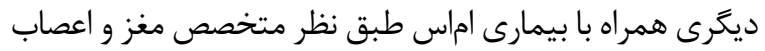

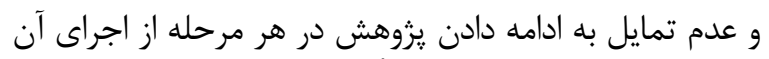

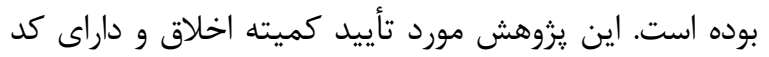

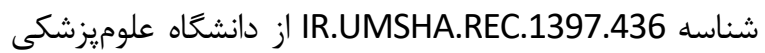
همدان است.

در اين مطالعه براى بدست آوردن حجم نمونه از فرمول شماره | (محاسبه حجم نمونه طبق فرمول كوكران) استفاده شد.

\section{$Z=1.96$}

$P=0.5, q=0.5$

$d=0.1$

بر اساس فرمول، حجم نمونه و9 نفر محاسبه شد. ابزارهاى

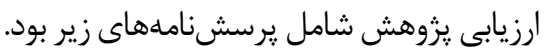

يرسشنامه اوقات فراغت بيماران اماس: توسط حسينى و

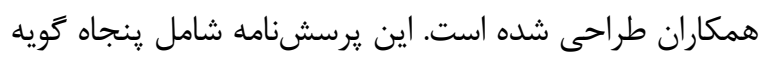

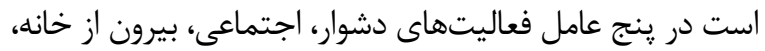

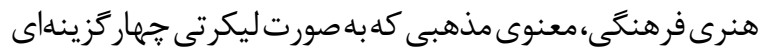

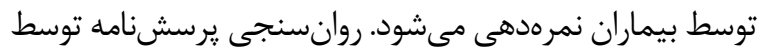

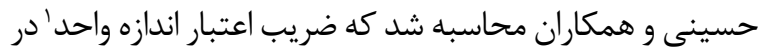

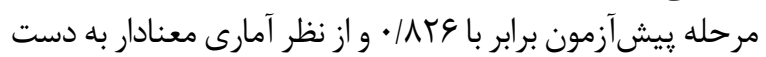

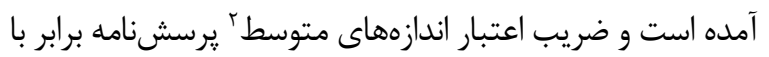

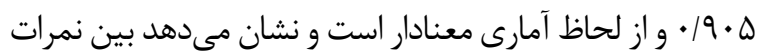

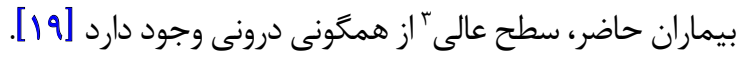
يرسشنامه افسردگى، اضطراب، استرس DASS: مقياس 
جدول ا. اطلاعات جمعيتشناختى

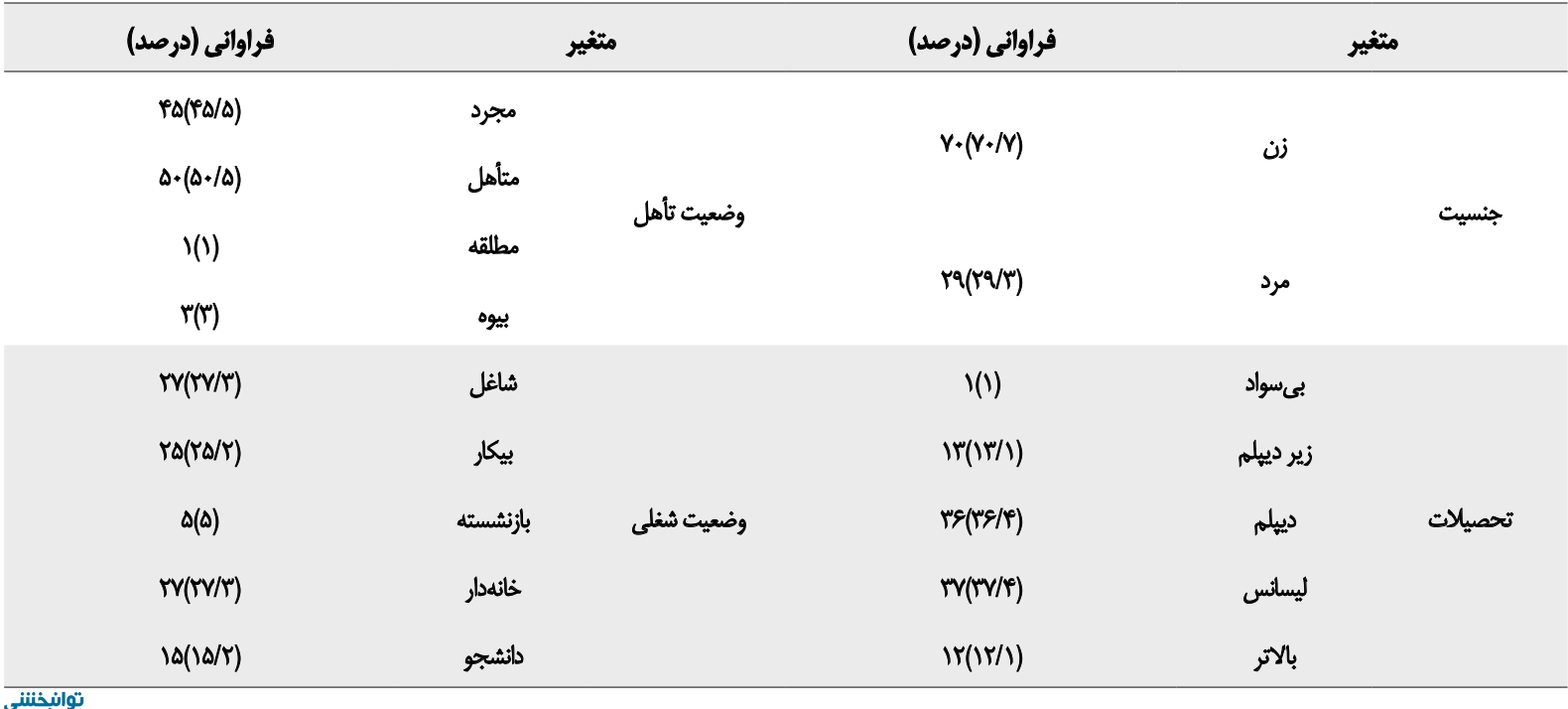

رابطه معنادارى را نشان داد. اوقات فراغت با سطح ناتوانى فرد،

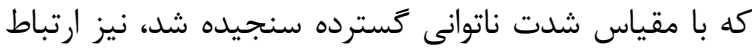

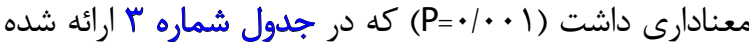

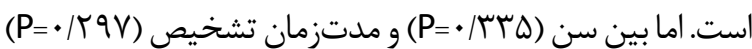
با اوقات فراغت رابطه معنادارى مشاهده نشد.

براى بررسى دقيقتر، ارتباط هريك از ينج حيطه اوقات

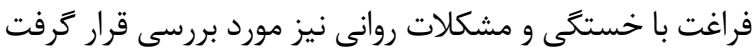

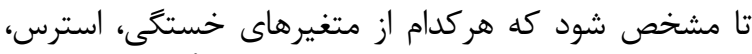

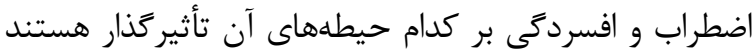

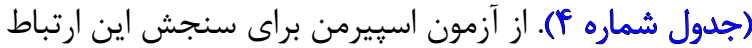

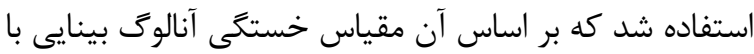

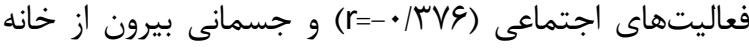

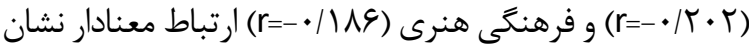

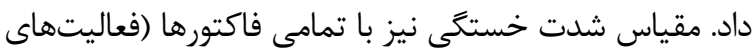

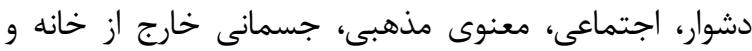

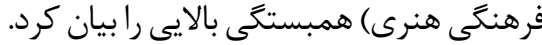

مشكلات روانى نيز با مقياس اضطراب افسردگى استرس

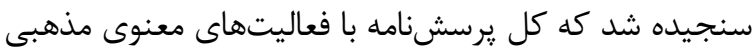

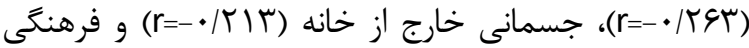

V. نشان داده شده است. تعداد شركت كنند

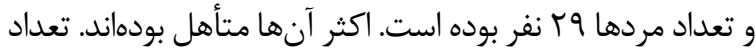

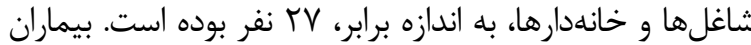

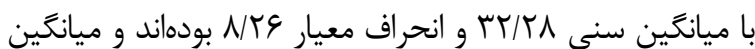

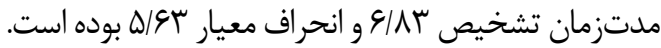

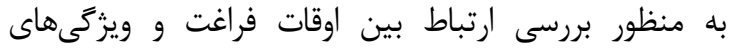

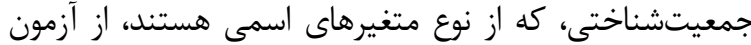

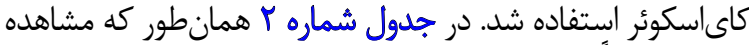

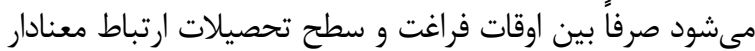

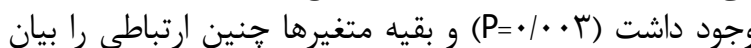
نكردند.

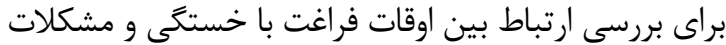

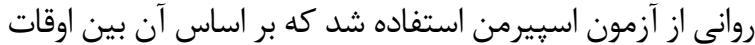

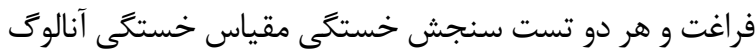

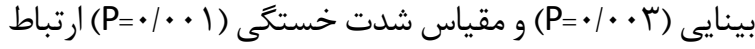

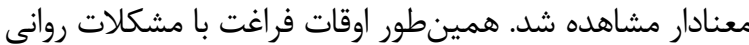

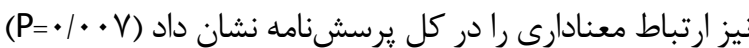

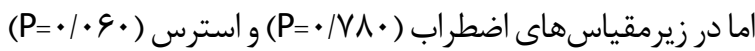

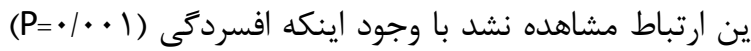

جدول r. ارتباط بين اوقات فراغت و ويثزتىهاى جمعيتشئاختى با آزمون كاياسكوئر

\begin{tabular}{|c|c|c|c|}
\hline معنادارى & ارزش & درجه أزادى & متغيرها \\
\hline . MPr & $\| v V \mid \cdot r$ & 109 & وضعيت أزدواج \\
\hline ./9४^ & $r r \cdot / \Delta T$ & res & وضعيت شغلى \\
\hline$t+t+r$ & $m / M$ & rir & سطح آموزش \\
\hline$. / \pi+q$ & $\Delta Q \triangle A$ & $\Delta r$ & جنسيت \\
\hline
\end{tabular}


جدول بـ ارتباط بين اوقات فراغت با متغيرهاي ئروهش (آزمون اسييرمن)

\begin{tabular}{|c|c|c|c|}
\hline معنادارى & ضريب همبستكي & تعداد & مثغير \\
\hline.$* \cdot r$ & $-\rightarrow$ Tra & 99 & حُستكى آثالوى ييناييى \\
\hline .1 .01 & $-+/ 2 \Delta$ & 99 & شدت خستكى \\
\hline .1 .9 & $-+/ 19$ & 99 & استرس \\
\hline . $/ \mathrm{A}$. & $-\cdot / 1 \gamma \lambda$ & 99 & اضطراب \\
\hline $.1 . .1$ &.$- / M T$ & 99 & اقسردمى \\
\hline 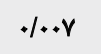 & $\rightarrow /$ Keq & 99 & ADSS \\
\hline $.1 . .1$ &.$- / 97 \Delta$ & 99 & EDSS \\
\hline.$/ \pi T \Delta$ & -.1 .24 & 99 & سن \\
\hline . /ray & $-+11+8$ & 99 & ملثتزمان تشيص \\
\hline
\end{tabular}

نمى كند كه مى تواند منطقى باشد. همه افراد در هر سنى تفريحات

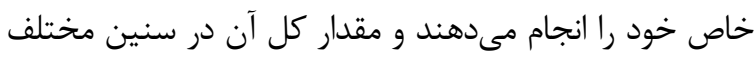

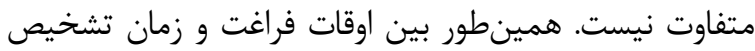

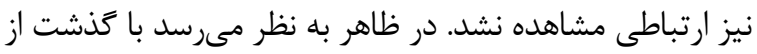

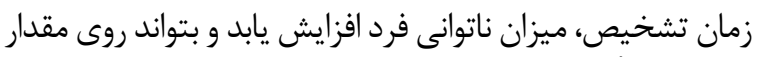

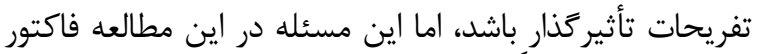

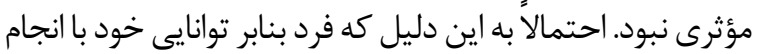

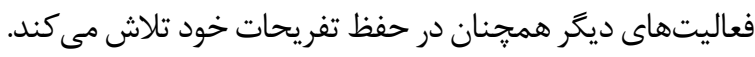
ديخر متغيرهاى جمعيتشناختى نيز بهجز سطح تحصيلات بر

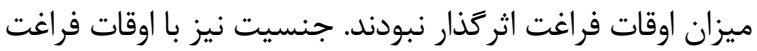

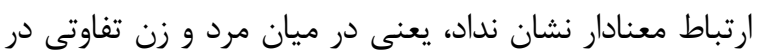

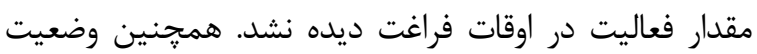
شغلى و وضعيت ازدواج فرد، تأثيرى بر ميزان اوقات فراغت فيده نيت همات

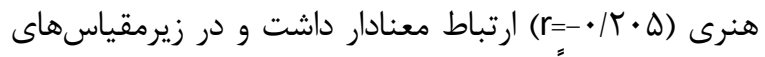

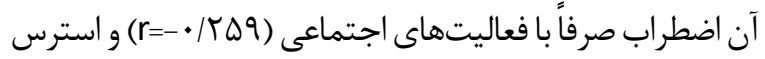

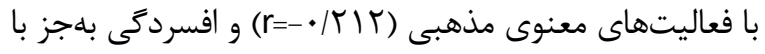

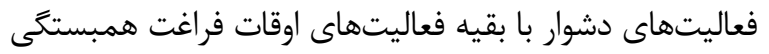

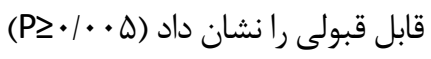

ç

يافتههاى يزوهش نشان مي دهد كه بر اساس آزمون اسيّيرمن

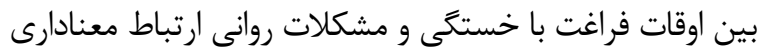

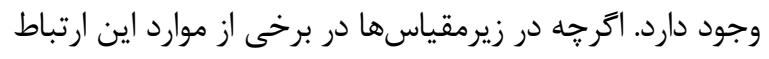

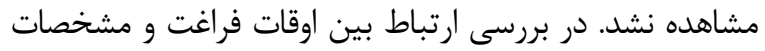

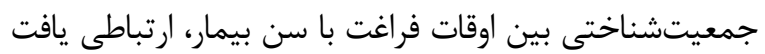

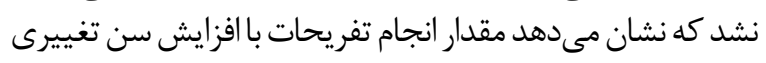

جدول ع. ارتباط بين ابعاد اوقات فراغت با خستكى و مشكلات روانيى

\begin{tabular}{|c|c|c|c|c|c|c|c|c|c|c|}
\hline \multicolumn{2}{|c|}{ فاكتوره } & \multicolumn{2}{|c|}{ فاكتورع } & \multicolumn{2}{|c|}{ فاكتورب } & \multicolumn{2}{|c|}{ ل فاكتورج } & \multicolumn{2}{|c|}{ فاكتور } & \multirow[b]{2}{*}{ متغير } \\
\hline معنادلري & معنادطحي & معناداري & معنادطحي & معناداريح & همبستئي & معنادطحي & ضمبستيكي & معنادطري & همبستيّي & \\
\hline$+1+\infty 1$ & $-+/ W E$ & $.1+40$ & $-+/ T+r$ &.$/ \mathrm{YA}$ & $-t / 1 r r$ & .10 .1 & $-+/$ Mq & (TH & $-t / 114$ & خستكى آنالوى بينايع \\
\hline 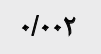 & $-+/ N+1$ & $.1 .+9$ &.$- / T \varepsilon$ & 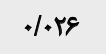 & $-\bullet / T M r$ & $\%$ & $-r / *$ & 1.40 &.$- /$ Tra & مقياس شدت خستكى \\
\hline$F \cdot M$ & $-* / / r r$ & $.1 . \Delta r$ & $-* / 198$ & .1 .40 & $-* / M I T$ &.$/ T A 1$ &.$- / 11 \Delta$ &.$/ 498$ &.$- / \cdot n^{\infty}$ & استرس \\
\hline. $\mid E 91$ & $-.1 .+4$ & $\%$ &.$- / M$ & $\% 1$. &.$- / T Q 9$ &.$/ 1 m$ & -./Iat & . &.$-|\cdot 1|$ & اضطراب \\
\hline.$/ 0.4$ & $-\cdot / r \cdot r$ & $.1 .+5$ &.$- / T A Y$ & 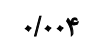 & $-* / 419$ & $.1+4$ & $-Y / .$. & $.1 . \Delta r$ &.$- / 19$ & |فسردكى \\
\hline.$/ .4 T$ & $-+/ r+\Delta$ & 1.40 & $-* / r \mid r$ & $.1 .+9$ & - & 1.8. &.$- / 19$ &.$/ M T$ &.$- / 1 \Delta Y$ & ADSS \\
\hline .1 .01 &.$- / A T Q$ & $.1 . .1$ & $-\cdot N \cdot P^{e}$ & $.1 . .1$ &.$- /$ req & .10 .1 & 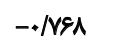 & $.1 . .1$ &.$-|M|$ & EDSS \\
\hline
\end{tabular}




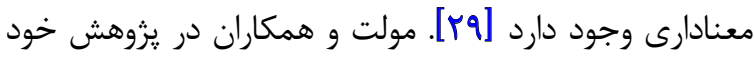

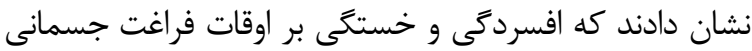

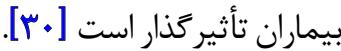

يافتههاى يزوهش استرود نشان مىدهد اوقات فراغت با بات

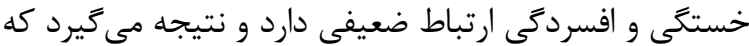

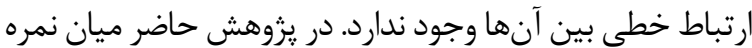

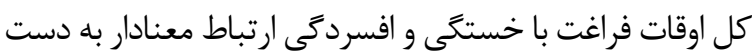

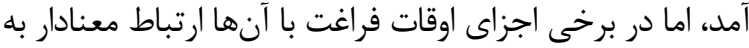

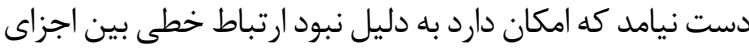

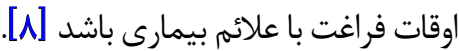
استفنز و همكاران در مطالعهاى طولى نشان دادند كه داشتن اوقات فراغت متوسط تا شديد در طول زندكى مى تواند

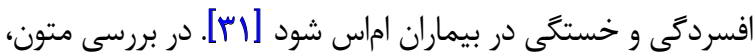

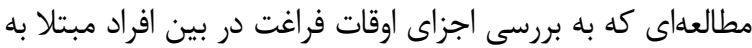

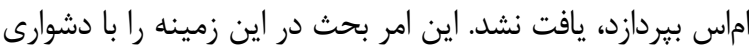

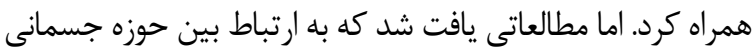

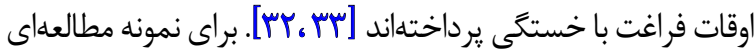

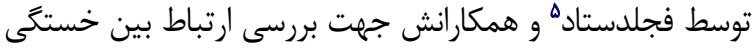

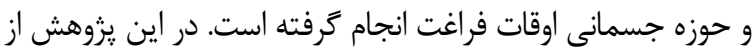

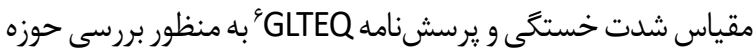

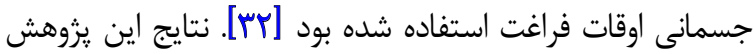

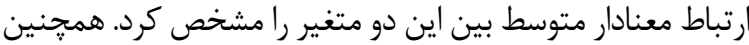

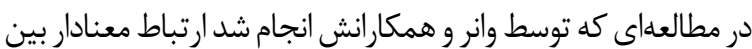

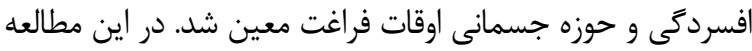

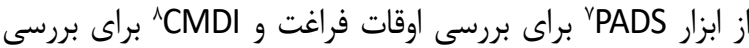

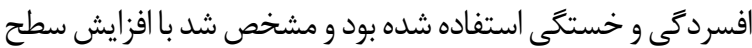

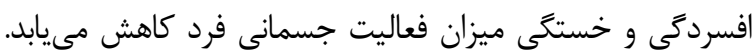

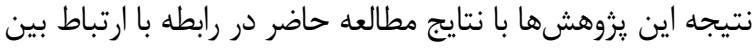

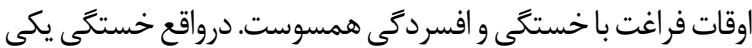

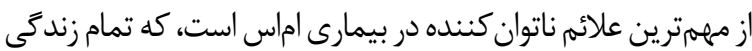

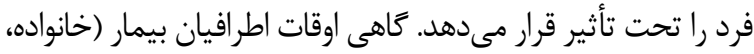

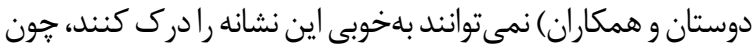

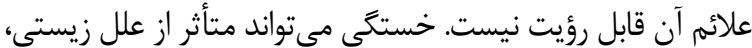

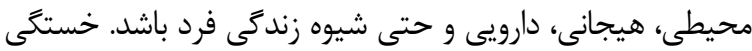

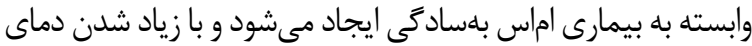

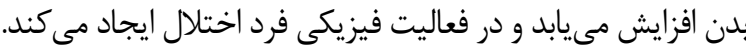

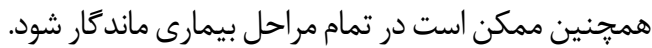

$$
\text { نتيجلَ'يرى }
$$

بر اساس يافتههاى مطالعه مىتوان نتيجه كرفت كه ميان

\section{Fjeldstad}

6. Godin Leisure-Time Exercise Questionnaire

7. Physical Activity Disability Scale

8. Chicago Multiscale Depression Inventory
ندارد. اين يعنى بيمار خه خانهدار و جه داراى شغل شئ و ويا بيكار

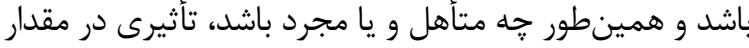

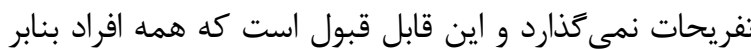

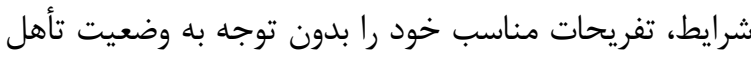

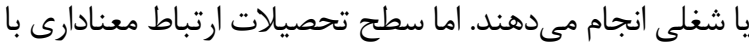

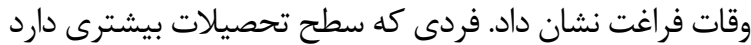

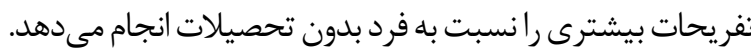

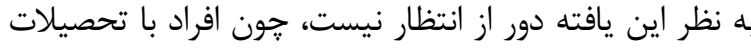

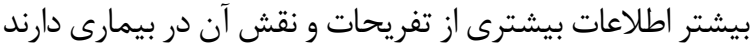

$$
\text { و بيشتر به آن مى يردازند. }
$$

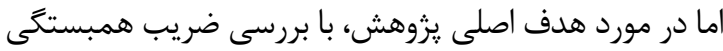

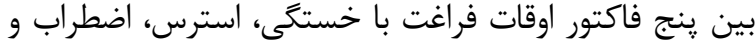

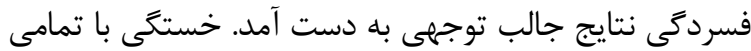

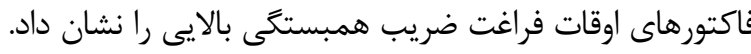

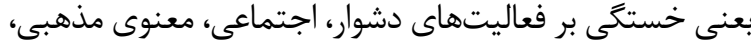

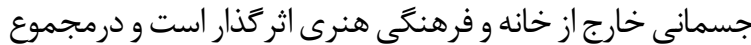

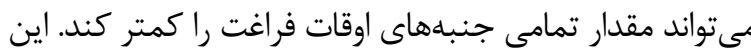

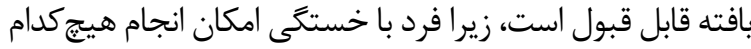

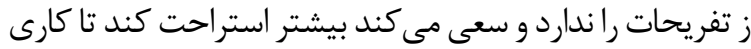

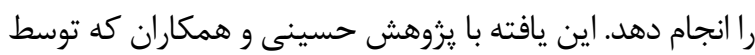

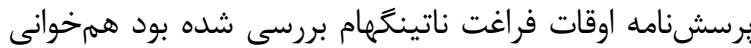

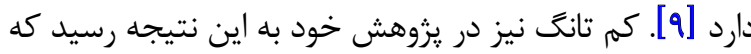

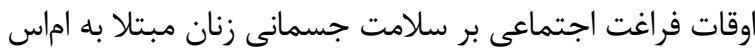

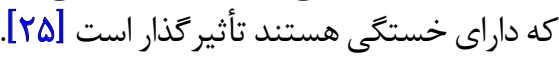
تأير استرس فقط بر جنبه فعاليتهاى اجتماعى اوقات فراغت

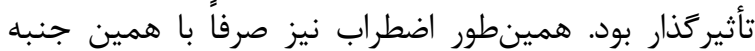

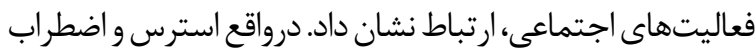

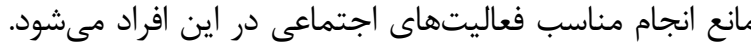

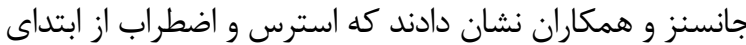

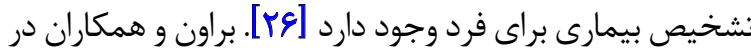

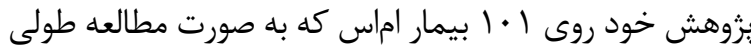

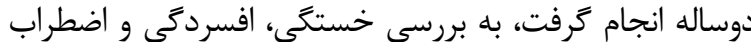

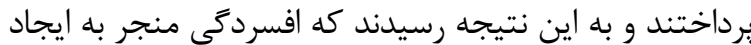

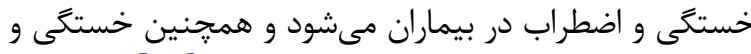

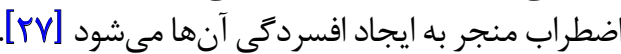
اما افسردگىاى كه در اكثر بيماران اماس ديده ميشود

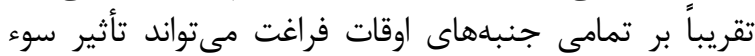

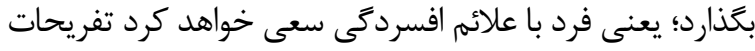

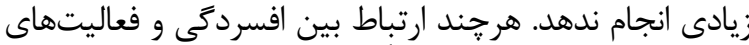

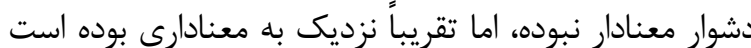
فرات

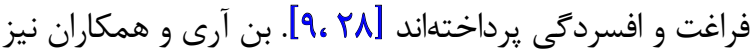

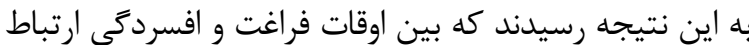


اوقات فراغت با مشكلات روانى و خستخى افر اد مبتلا به مولتيبل

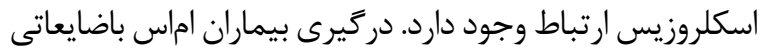

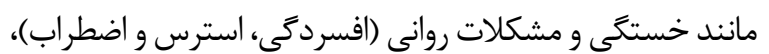

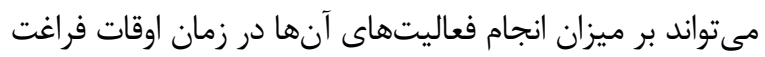
موثر باشد و مقدار آن را كاهش دهد.

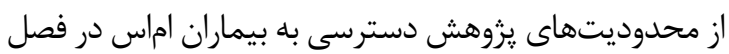

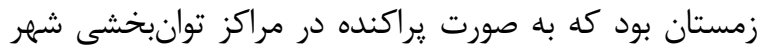

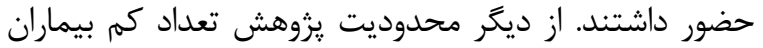

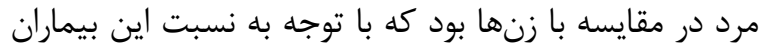

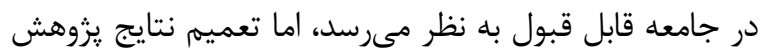

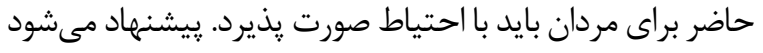

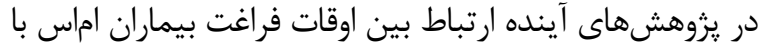

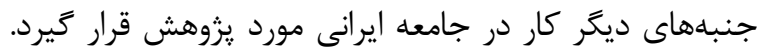

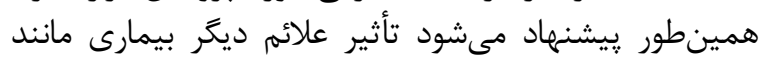

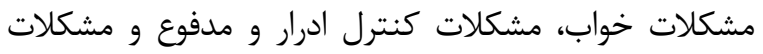
شناختى بر اوقات فراغت اين بيماران مورد بررسى قرار مرات كيرد.

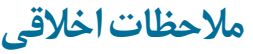

$$
\text { يبيروى از اصول اخلاق يثوهش }
$$

اين ملاحظات توسط كميته اخلاق دانشكاه علوميزشكى همدان با شماره IR.UMSHA.REC.1397,436 به ثبت راح رسيده

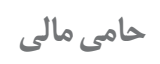

اين مقاله بركرفته از طرحى تحقيقاتى با شماره ثبت qV.ATYFAFF علوميزشكى همدان بوده است.

$$
\text { مشاركت نويسند }
$$

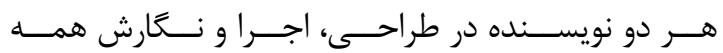

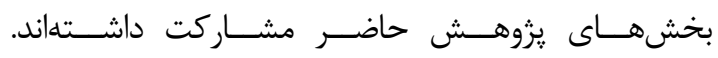

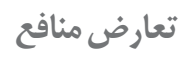

بنابر اظهار نويسندكان اين مقاله تعارض منافع ندارد. 


\section{Referencs}

[1] Mozo-Dutton L, Simpson J, Boot J. MS and me: Exploring the impact of multiple sclerosis on perceptions of self. Disability and Rehabilitation. 2012; 34(14):1208-17. [DOI:10.3109/09638288.201 1.638032

[2] Statistical Center of Iran. Vice presidency for strategic planning and supervision statistical center of Iran [Internet]. 2011 [Updated 2011]. Available from: https://www.amar.org.ir/Portals/1/Iran/ Atlas_Census_2011.pdf

[3] Etemadifar M, Maghzi AH. Sharp increase in the incidence and prevalence of multiple sclerosis in Isfahan, Iran. Multiple Sclerosis Journal. 2011; 17(8):1022-7. [DOI:10.1177/1352458511401460]

[4] Burks JS, Bigley GK, Hill HH. Rehabilitation challenges in multiple sclerosis. Annals of Indian Academy of Neurology. 2009; 12(4):296-306. [DOI:10.4103/0972-2327.58273]

[5] Radomski MV, Trombly Latham CA, editors. Occupational therapy for physical dysfunction. Philadelphia: Wolters Kluwer Health; 2008. https://books.google.com/books?id=uZ155EfugoC\&source $=$ gbs_navlinks_s

[6] Kos D, Kerckhofs E, Nagels G, D'hooghe MB, Ilsbroukx S. Origin of fatigue in multiple sclerosis: Review of the literature. Neurorehabilitation and Neural Repair. 2008; 22(1):91-100. [DOI:10.1177/1545968306298934]

[7] Trojan DA, Arnold D, Collet JP, Shapiro S, Bar-Or A, Robinson A, et al. Fatigue in multiple sclerosis: Association with disease-related, behavioural and psychosocial factors. Multiple Sclerosis Journal. 2007; 13(8):985-95. [DOI:10.1177/1352458507077175]

[8] Stroud NM, Minahan CL. The impact of regular physical activity on fatigue, depression and quality of life in persons with multiple sclerosis. Health and Quality of Life Outcomes. 2009; 7:68. [DOI:10.1186/1477-7525-7-68]

[9] Hosseini SMS, Rassafiani M, Mazdeh M, Haghgoo HA, Nurani Gharaborghe S. [Effect of fatigue, depression and cognitive dysfunction on participation in leisurely activities among people with multiple sclerosis (Persian)]. Middle Eastern Journal of Disability Studies. 2017; 7:36. http://jdisabilstud.org/article-1-585-en.html

[10] Keshkar S, Ehsani M, Koozechian H, Ghasemi H, Mohammad S. Examining the hierarchical model of leisure constraints among women in Tehran regarding sports participation. International Journal of Sport Studies. 2012; 2(11):561-70. https://www.researchgate. net/profile/Sardar-Mohammadi/publication/247777361

[11] Arab-Moghaddam N, Henderson KA, Sheikholeslami R. Women's leisure and constraints to participation: Iranian perspectives. Journal of Leisure Research. 2007; 39(1):109-26. [DOI:10.1080/00222216. 2007.11950100]

[12] Ghaem H, Mohammad Salehi N, Mohammad Beigi A. [Assessment of spending leisure time in students of Shiraz University of Medical Sciences, 2005 (Persian)]. Iranian Journal of Medical Education. 2008; 8(1):71-80. http://pdfarchive.ir/pack-01/ Do_62713871908.bak.pdf

[13] Tondnevis F. [The status of physical activities at leisure time of iranian residence (Persian)]. Research on Sport Science. 2003; 1(4):11533. https://www.sid.ir/fa/journal/ViewPaper.aspx?ID=9301
[14] Tondnevis F. [The physical acitivities in leisure time of the iranian women (Persian)]. Harakat. 2002; 12(12):87-104. https:// joh.ut.ac.ir/article_10360.html

[15] Fattahi Masrour F, Tondnevis F, Mozaffari AA. Investigating of leisure time activities in female students at Iran's Islamic Azad University. European Journal of Experimental Biology. 2012; 2(4):1062-70. https://www.imedpub.com/articles/investigatingof-leisure-time-activities-in-female-students-at-irans-islamicazaduniversity.pdf

[16] Saraii MH, Roosta M, Oshnoui A. [Factors affecting leisure in urban areas of Iran (Persian)]. Journal of Regional Planning. 2012; 2(7):25-37. http://jzpm.miau.ac.ir/article_51.html

[17] Chwastiak L, Ehde DM, Gibbons LE, Sullivan M, Bowen JD, Kraft GH. Depressive symptoms and severity of illness in multiple sclerosis: Epidemiologic study of a large community sample. American Journal of Psychiatry. 2002; 159(11):1862-8. [DOI:10.1176/appi.ajp.159.11.1862]

[18] Prejza S. The significance of leisure among persons diagnosed with multiple sclerosis [MSc. thesis]. San Jose, CA: San Jose State University; 1997. [DOI:10.31979/etd.2xnk-m7y9]

[19] Hosseini SMS, Sarhady M, Nurani Gharaborghe S, Mazdeh M. [Leisure of people with Multiple Sclerosis: A content analysis (Persian)]. Iranian Rehabilitation Journal. 2017; 15(1):23-30. http://irj.uswr.ac.ir/article-1-609-en.htm

[20] Lovibond SH, Lovibond PF. Manual for the depression anxiety stress scales. Sydney: Psychology Foundation of Australia; 1996. https://books.google.com/books?id=mXoQHAAACAAJ\&dq

[21] Parkitny L, McAuley J. The Depression Anxiety Stress Scale (DASS). Journal of Physiotherapy. 2010; 56(3):204. [DOI:10.1016/S1836-9553(10)70030-8]

[22] Samani S, Jokar B. [Investigation of validity and reliability of short form of depression, anxiety and stress scale (Persian)]. Journal of Social Sciences and Humanities, Shiraz University. 2007; 26(3):65-77. https://www.sid.ir/Fa/Journal/ViewPaper. aspx?id $=82319$

[23] Krupp LB, LaRocca NG, Muir-Nash J, Steinberg AD. The fatigue severity scale: Application to patients with multiple sclerosis and systemic lupus erythematosus. Archives of Neurology. 1989; 46(10):1121-3. [DOI:10.1001/archneur.1989.00520460115022]

[24] Azimian M, Shahvarughi Farahani A, Dadkhah A, Fallahpour M, Karimlu M. Fatigue severity scale: The psychometric properties of the Persian-version in patients with multiple sclerosis. Research Journal of Biological Sciences. 2009; 4(9):974-7. https:// medwelljournals.com/abstract/?doi=rjbsci.2009.974.977

[25] Khemthong S, Packer TL, Passmore A, Dhaliwal SS. Does social leisure contribute to physical health in multiple sclerosis related fatigue. Annual in Therapeutic Recreation. 2008; 16:71-80. https://www.researchgate.net/publication/46165386

[26] Janssens ACJW, van Doorn PA, de Boer JB, van der Meché FGA, Passchier J, Hintzen RQ. Impact of recently diagnosed multiple sclerosis on quality of life, anxiety, depression and distress of patients and partners. Acta Neurologica Scandinavica. 2003; 108(6):389-95. [DOI:10.1034/j.1600-0404.2003.00166.x] 
[27] Brown RF, Valpiani EM, Tennant CC, Dunn SM, Sharrock M, Hodgkinson S, et al. Longitudinal assessment of anxiety, depression, and fatigue in people with multiple sclerosis. Psychology and Psychotherapy: Theory, Research and Practice. 2009; 82(1):41-56. [DOI:10.1348/147608308X345614]

[28] Tauil CB, Grippe TC, Dias RM, Dias-Carneiro RPC, Carneiro NM, Aguilar ACR, et al. Suicidal ideation, anxiety, and depression in patients with multiple sclerosis. Arquivos de Neuro-Psiquiatria. 2018; 76(5):296-301. [DOI:10.1590/0004-282x20180036]

[29] Ben Ari E, Johansson S, Ytterberg Ch, Bergström J, von Koch L. How are cognitive impairment, fatigue and signs of depression related to participation in daily life among persons with multiple sclerosis? Disability and Rehabilitation. 2014; 36(23):2012-8. [DO I:10.3109/09638288.2014.887797]

[30] Motl RW, McAuley E, Snook EM, Gliottoni RC. Physical activity and quality of life in multiple sclerosis: Intermediary roles of disability, fatigue, mood, pain, self-efficacy and social support. Psychology, Health \& Medicine. 2009; 14(1):111-24. [DOI:10.1080/13548500802241902]

[31] Stephens S, Shams Sh, Lee J, Grover SA, Longoni G, Berenbaum $\mathrm{T}$, et al. Benefits of physical activity for depression and fatigue in multiple sclerosis: A longitudinal analysis. The Journal of Pediatrics. 2019; 209:226-32.E2. [DOI:10.1016/j.jpeds.2019.01.040]

[32] Fjeldstad C, Brittain DR, Fjeldstad AS, Pardo G. Fatigue and thermo sensitivity affect physical activity in multiple sclerosis. The Journal of Applied Research. 2010; 10(3):108-15. https://www. researchgate.net/publication/234038090

[33] Vanner EA, Block P, Christodoulou CC, Horowitz BP, Krupp LB. Pilot study exploring quality of life and barriers to leisuretime physical activity in persons with moderate to severe multiple sclerosis. Disability and Health Journal. 2008; 1(1):58-65. [DOI:10.1016/j.dhjo.2007.11.001 